\title{
Radioisotope Distribution Program Progress Report for April 1976
}

\author{
E. Lamb
}




\section{DISCLAIMER}

This report was prepared as an account of work sponsored by an agency of the United States Government. Neither the United States Government nor any agency Thereof, nor any of their employees, makes any warranty, express or implied, or assumes any legal liability or responsibility for the accuracy, completeness, or usefulness of any information, apparatus, product, or process disclosed, or represents that its use would not infringe privately owned rights. Reference herein to any specific commercial product, process, or service by trade name, trademark, manufacturer, or otherwise does not necessarily constitute or imply its endorsement, recommendation, or favoring by the United States Government or any agency thereof. The views and opinions of authors expressed herein do not necessarily state or reflect those of the United States Government or any agency thereof. 


\section{DISCLAIMER}

Portions of this document may be illegible in electronic image products. Images are produced from the best available original document. 
Printed in the United States of America. Available from National Technical Information Service

U.S. Department of Commerce

5285 Port Royal Road, Springfield, Virginia 22161

Price: Printed Copy $\$ 3.50$; Microfiche $\$ 2.25$

This report was prepared as an account of work sponsored by the United States Government. Neither the United States nor the Energy Research and Development Administration/United States Nuclear Regulatory Commission, nor any of their employees, nor any of their contractors, subcontractors, or their employees, makes any warranty, express or implied, or assumes any legal liability or responsibility for the accuracy, completeness or usefulness of any information, apparatus, product or process disclosed, or represents that its use would not infringe privately owned rights 
Contract No. W-7405-eng-26

OPERATIONS DIVISION

\section{RADIOISOTOPE DISTRIBUTION PROGRAM \\ PROGRESS REPORT FOR APRIL 1976}

This report wes prepared as an socount of work Ths report whs prepared as an cocount of work the United States nor the United States Energy Research and Development Administ ration, not any of their employees, nor any of their contractors. subcontractors, os their employeas, makes any wamenty, express or implied, or assumes any lega

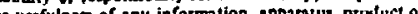
or useluliness of any information, spparatus, procuct or process disciosed, or represents
infringe privately owned rights.

\author{
E. Lamb
}

Work Sponsored by

ERDA Division of Biomedical and Environmental Research

\title{
JULY 1976
}

NOTICE This document contains informetion of a preliminary nature and was prepared primarily for internal use at the Oak Ridge National Laboratory. It is subject to revision or correction and therefore does not represent a final report.

OAK RIDGE NATIONAL LABORATORY 
THIS PAGE

\section{WAS INTENTIONALLY \\ LEFT BLANK}


CONTENTS

$\underline{\text { Page }}$

RADIOISOTOPE PRODUCTION AND MATERIALS DEVELOPMENT. • • • • .

Reactor Products Pịlot Production............ 1 ACCELERATOR-PRODUCED ISOTOPES. . . . . ........ 1

Cyclotron Products Pilot Production. . . . . . . . 1

FISSION PRODUCTS . . . . . . . . . . . . . . 2

Krypton-85 Enrichment Facility . . . . . . . . 2

Cesium-137 Pilot Production. . . . . . . . . . . 2

Strontium-90 Pilot Production. ............ 3

Short-Lived Fission Production . . . . . . . . . 4

RADIOISOTOPE SALES . . . . . . . . . . . . . . . 5

PUBLICATIONS ..................... . . . . . 5

REPORTS......................... . 5 
RADIOISOTOPE DISTRIBUTION PROGRAM

PROGRESS REPORT FOR APRIL 1976

$$
\text { E. Lamb }
$$

RADIOISOTOPE PRODUCTION AND MATERIALS DEVELOPMENT

REACTOR-PRODUCED RADIOISOTOPES

Reactor Products Pilot Production ( $R$. W. Schaich)

(Production and Inventory Accounts)

$\frac{\text { Processcd Units }}{\text { Radioisotope }}$
Calcium-47

\section{ACCELERATOR-PRODUCED ISOTOPES}

Cyclotron Products Pilot Production (M. R. Skidmore)

(Production and Inventory Accounts)

Apri.1 1976 ORNL 86-Inch Cyclotron runs for ORNL and non-ORNL programs are given in Table 1 。

Table 1。 Cyclotron Irradiations and Runs for April 1976

\begin{tabular}{|c|c|c|c|c|c|}
\hline Date & Customer & Product & Target & $\begin{array}{l}\text { Total Time } \\
\text { (hr:min). }\end{array}$ & $\begin{array}{c}\text { Total } \\
\text { Charges }\end{array}$ \\
\hline$\because$, & · & ORNL Programs & & & \\
\hline \multirow[t]{3}{*}{$\begin{array}{l}4-5-76 \\
4-13-76 \\
4-19-76\end{array}$} & $\begin{array}{l}\text { ORAU } \\
\text { ORAU } \\
\text { ORAU }\end{array}$ & $\begin{array}{l}\text { Carbon }-11 \\
\text { Carbon }-11 \\
\text { Carbon-11 }\end{array}$ & $\begin{array}{l}\text { Boron Oxide } \\
\text { Boron Oxide } \\
\text { Boron Oxlde }\end{array}$ & $\begin{array}{r}5: 15 \\
5: 15 \\
5: 15 \\
\end{array}$ & $\begin{array}{l}642 \\
642 \\
642 \\
\end{array}$ \\
\hline & & & & $.15: 45$ & $\$ 1,926$ \\
\hline & No & n-ORNL Progra & & & \\
\hline \multirow[t]{3}{*}{$\begin{array}{l}4-2-76 \\
4-17-76 \\
4-23-76\end{array}$} & $\begin{array}{l}\text { New England Nuclear } \\
\text { New England Nuclear } \\
\text { International Chemi- } \\
\text { cal \& Nuclear }\end{array}$ & $\begin{array}{l}\text { Cobalt-57 } \\
\text { Gallium-67 } \\
\text { Cobalt-57 }\end{array}$ & $\begin{array}{l}\text { Nicke1-58 } \\
\text { Zinc-68 } \\
\text { Nicke1-58 }\end{array}$ & $\begin{array}{r}51: 15 \\
8: 15 \\
49: 15 \\
\end{array}$ & $\begin{array}{r}9,031 \\
1,440 \\
9,631 \\
\end{array}$ \\
\hline & & & & $108: 45$ & $\$ 20,102$ \\
\hline & Isotop & es Sales Inve & ory & & \\
\hline \multirow[t]{2}{*}{$\begin{array}{l}4-9-76 \\
4-15-76\end{array}$} & $\begin{array}{l}\text { Isotopes Sales } \\
\text {-Isotopes Sales }\end{array}$ & $\begin{array}{l}\text { Cobalt }-57 \\
\text { Bismuth-207 }\end{array}$ & $\begin{array}{l}\text { Nickel } \\
\text { Lead }\end{array}$ & $\begin{array}{r}57: 15 \\
9: 15 \\
\end{array}$ & $\begin{array}{r}9,447 \\
1,733 \\
\end{array}$ \\
\hline & & & & $66: 30$ & $\$ 11,180$ \\
\hline
\end{tabular}




\section{Cyclotron Operations}

Operations were smooth this month. A teflon insulator on the flat plate dolly, which had shorted due to radiation damage, was replaced. The filament in the ion source was replaced; filament had operated 219 hours.

FISSION PRODUCTS

Krypton-85 Enrichment Facility. (F. N. Case)

Transfer of the enriched ${ }^{85} \mathrm{Kr}$ product was begun on April 2, 1976 from column $A B$. Three transfers were successfully made. During the fourth transfer a release of enriched krypton occurred allowing $~ 30$ curies of $7.5 \%{ }^{85} \mathrm{Kr}$ to escape from a rubber tubing to copper tubing connection. Al1 unload1ng operations have ceased until investigation of cause and making of equipment modifications to prevent the occurrence and reduce the possibility of personnel exposure are complete: No transfers of material have been made since that date.

On April 17, 1976 the colums shut down automatically due to an overtemperature. Investigation showed that a flexible conduit containing power lines to the compressors in the chilled water system wore through a copper refrigerant line due to vibration of the compressor system. The loss of refrigerant resulted in automatic compressor shutdown and a subsequent rise in colum temperature. The system was repaired and put back: in service on April 21, 1976:

Cesium-137 Pilot Production (R. W. Schaich)

(Production and Inventory Accounts)

\section{Process Status.}

The analytical data on the purified powder batches is not complete. Resampling of the last four batches may be required to quantify the total ${ }^{137} \mathrm{Cs}$ recovered。 The cesium analysis of the first batch showed $50 \% 133 \mathrm{Cs}$, $14 \%{ }^{135} \mathrm{Cs}$, and $36 \%{ }^{137} \mathrm{Cs}$.

2. Operational Summary

\section{Product - Inventory}

(Decay calculated through April 30, 1975)

\section{Inventory Material}

In-Process

Cesium-137 chloride powder

Special form cans and Fabricated Sources
Amount (Ci) 
Non-Inventory Material

Amount (Ci)

Material returned or stored for customer

Puerto Rico sources

Lockheed

AECL powder

Radiation Resources

Minn. Mining \& Mfg. Company

Gamina Industries

J. L。 Shepherd

8,200

20,300

73,800

34,600

9,500

8,600

Total Non-Inventcry Material

13,400

\begin{abstract}
Total Non-Inventcry Material
\end{abstract}
TOTAL INVENTORY AND NON-INVENTORY MATERIAL

168,400

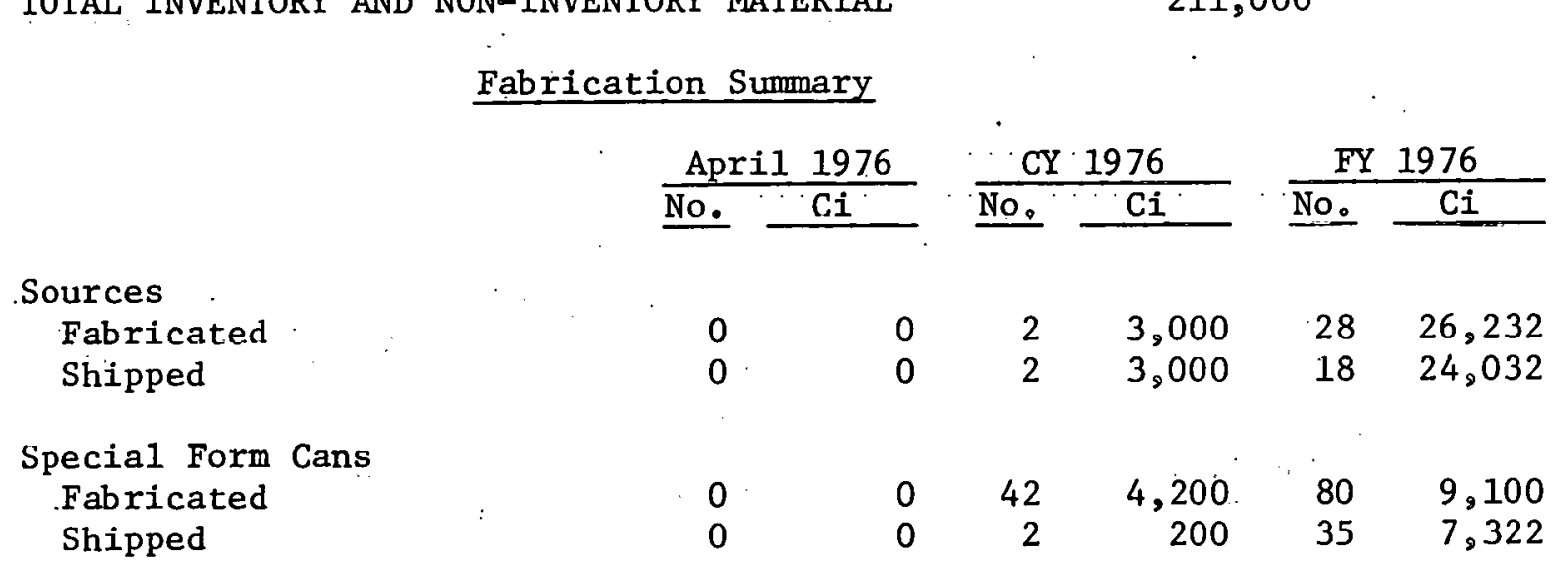

3. Current . Orders

All orders on hand have been completed and the material placed into storage awaiting receipt of release for the material.

Strontium-90 Pilot Production (R. W. Schaich)

(Production and Inventory Accounts)

1. Process: Status

The ${ }^{90} \mathrm{Sr}$ process and manipulator cells are being decontaminated under the ERDA Decommissioning Program. The ${ }^{0} \mathrm{Sr}$ powder was removed from the FPDL, encapsulated, and stored for future orders.

\title{
Product Inventory
}

(Decay calculated through April 30, 1975)

Inventory Material

${ }^{90} \mathrm{Sr}$ titanate powder $( \pm 5 \%)$

Sources in fabrication

RCA source

${ }^{90} \mathrm{Sr}$ silicate powder

stock powder cans
Amount (Ci)

$$
\begin{array}{r}
476,000 \\
0 \\
57,800 \\
28,200 \\
4,100 \\
\hline 566,100 \\
\hline
\end{array}
$$

Total Inventory Material. 
Non-Inventory Material

FPDL recovery material

Quehanna recovery materia1

Weather Bureau source

SNAP-7B

SNAP $-7 C$

SNAP-7D

SNAP material purchase ${ }^{a}$
Amount (Ci)

18,200

44,400

11,800

161,500

25,400

147,800

$\underline{256,600}$

665,700

$1,231,800$

TOTAL INVENTORY AND NON-INVENTORY MATFRTAT.

atront1um-90 purchased under DRRD program.

\section{Fabrication Summary}

$\frac{\text { April } 1976}{\text { No. } \frac{\mathrm{Ci}}{4}}$

$\frac{\mathrm{CY} 1976}{\mathrm{No} \cdot \mathrm{Ci}}$

$\frac{\text { FY } 1976}{\text { No. } \frac{\mathrm{Ci}}{}}$

Sources
Fabricated
Shipped

Sperial Form Cans

Fabricated

Shipped $\begin{array}{lll}0 & 0 & 0 \\ 0 & 0 & 0\end{array}$

0

0

0
4

0
1.34

$0 \quad 0$

0

0

Short-Lived Fission Production (R. W. Schaich)

(Production and Inventory. Accounts)

Isntope

Xenon-133

Yttrium-91

Barium-140

Strontium-89

Ruthenium-103
Number of Batchco

2

1

1 .

1

1
AniưunL (CL)

700

21

1

3 


\title{
RADIOISOTOPE SALES
}

\author{
J.E.Ratledge
}

Shipments made during the month that may be of interest are listed below:

Customer

Isotope

Amount

\section{Large Quantities}

American Atomics

New England Nuclear Corporation

Schwarz/Mann

Self-Powered Lighting

U. S。 Radium Corporation
Tritium
Tritium
Tritium
Tritium
Tritium

Withdrawn Items

Iodine-131

$5 \mathrm{mCi}$

Items Used in Cooperative Programs

ORAU

Dysprosium-157

$40 \mathrm{mCi}$

The radioisotope sales and shipments for the first ten months of FY 1975 and FY 1976 are given in Table 2.

Table 2. Radioisotope Sales and Shipments

\begin{tabular}{|c|c|c|c|c|}
\hline Item & \multicolumn{2}{|c|}{$\begin{array}{l}7-1-74 \text { thru } \\
4-30-75\end{array}$} & \multicolumn{2}{|c|}{$\begin{array}{c}7-1-75 \text { thru } \\
4-30-76\end{array}$} \\
\hline $\begin{array}{l}\text { Inventory items } \\
\text { Majur products. } \\
\text { Radioisotope services } \\
\text { Cyclotron irradiations } \\
\text { Miscellaneous processed materials } \\
\text { Packing and Shipping }\end{array}$ & $\$$ & $\begin{array}{r}317,453 \\
84,375 \\
92,453 \\
84,257 \\
107,250 \\
62,520 \\
\end{array}$ & $\$$ & $\begin{array}{r}225,282 \\
42,600 \\
75,044 \\
142,080 \\
43,473 \\
86,288 \\
\end{array}$ \\
\hline Total & $\$$ & 748,308 & $\$$. & 614,767 \\
\hline Number of Shipwints & & 1,319 & & 1,369 \\
\hline
\end{tabular}

\section{PUBLICATIONS}

\section{REPORTS}

E. Lamb, Radioisotope Distribution Program Progress Report for March 1976, ORNL/TM-5469, Oak Kidge Nat1onal Laburatory (May 1976)。 
THIS PAGE

\section{WAS INTENTIONALLY LEFT BLANK}


ORNL/TM-5537

INTERNAL DISTRIBUTION

1. E. E. Beauchamp

2. T. A. Butler

3. F. N. Case

4. W. R. Casto

5. J. A. Cox

6. R. F. Hibbs

7. E. Lamb

8. H. H. Nichol

9. C. L. Ottinger

10. J. K. Poggenburg

11. H. Postma
12. M. E. Ramsey

13. J. E. Ratledge

14. C. R. Richmond

15. A. F. Rupp

16. R. W. Schaich

17. M. R. Skidmore

18. M. J. Skinner

19-20. Central Research Library

21-22. Laboratory Records Department

23. Laboratory Records - RC

24. Document Reference Section

EXTERNAL DISTRIBUTION

25. B. J. Dropesky, LASL, Los Alamos, New Mexico

26-27. J. H. Jarrett, PNL, Richland, Washington

28. D. K. Jones, Richland Operations Office, Richland, Washington

29. L. M. Knights, Atlantic Richfield Hanford Company, Richland, Washington

30. J. N. Maddox, ERDA-DBER, Washington, D. C.

31. W. E. Mott, ERDA-DBER, Washington, D. C.

32. H. A. O'Brien, LASL, Los Alamos, New Mexico

33. F. J. Skozen (Krizek), Argonne Cancer Research Hospital, Chicago

34. L. G. Stang, Jr., BNL, New York

35-36. R. W. Wood, ERDA-DBER, Washington, D. C.

37. Donner Laboratory Library, University of California, Berkeley, Calif., 94720

38. Research and Technical Support Division, ORO

39-40. Technical Information Center 